\title{
A METHOD FOR AUTOMATIC EXTRACTION OF MODEL PARAMETERS FROM FUNDAMENTAL FREQUENCY CONTOURS OF SPEECH
}

\author{
Shuichi Narusawa ${ }^{1}$, Nobuaki Minematsu ${ }^{1}$, Keikichi Hirose ${ }^{2}$ and Hiroya Fujisaki ${ }^{3}$ \\ ${ }^{1}$ Graduate School of Information Science and Technology, University of Tokyo \\ ${ }^{2}$ Graduate School of Frontier Sciences, University of Tokyo ${ }^{3}$ Prof. Emeritus, University of Tokyo \\ 7-3-1, Hongo, Bunkyo-ku, Tokyo, 113-8656, JAPAN \\ \{narusawa, mine, hirose, fujisaki\}@ gavo.t.u-tokyo.ac.jp
}

\begin{abstract}
The process of generating the $F_{0}$ contour of speech has been modeled quite accurately in mathematical terms by Fujisaki and his coworkers, but the extraction of parameters of the underlying commands from an observed $F_{0}$ contour is an inverse problem that can be solved only by successive approximation. In order to guarantee an efficient and accurate search for the solution, one needs to start with a set of initial values that are close enough to the optimum. This paper presents a method for pre-processing a measured $F_{0}$ contour to obtain its approximation consisting of thirdorder polynomial segments that are continuous and differentiable everywhere. It is shown that the proposed method allows one to obtain first-order approximations to the parameters of accent commands for about $90 \%$ of all the accent commands, and of phrase commands for about $84 \%$ of all the phrase commands.
\end{abstract}

\section{INTRODUCTION}

The contour of the voice fundamental frequency (henceforth $F_{0}$ contour) plays an important role in expressing information on the prosody of an utterance, i.e., the information concerning the lexical tone/accent, syntactic structure, and discourse focus. As it is well known, an $F_{0}$ contour generally consists of slowly-varying components corresponding to phrases and clauses and rapidly-varying components corresponding to word accents or syllable tones. The exact relationships between these components of an $F_{0}$ contour and the underlying linguistic information have been formulated by Fujisaki and his coworkers [1], and expressed as a model for the process of $F_{0}$ contour generation. It has been widely shown that the model can generate very close approximations to observed $F_{0}$ contours from a relatively small number of parameters representing the linguistic information, and is therefore quite useful in speech synthesis.

While it is quite straightforward to derive an $F_{0}$ contour from a set of model parameters, the inverse problem, i.e., the derivation of model parameters from a given $F_{0}$ contour, cannot be solved analytically, but can be solved only by the method of successive approximation. Unless one does not start with a good first-order approximation, however, successive approximations tend to be quite inefficient, and may not guarantee convergence to a true solution. The present paper describes a method for finding a good firstorder approximation for the set of model parameters from an observed $F_{0}$ contour. Although the method is applicable, with certain language-specific modifications, to $F_{0}$ contours of various languages, the present paper deals with $F_{0}$ contours of Japanese.

\section{A MODEL FOR THE GENERATION PROCESS OF $\boldsymbol{F}_{0}$ CONTOURS OF JAPANESE UTTERANCES}

Figure 1 shows the model for the process of generation of $F_{0}$ contours of Japanese utterances. The mechanism that produces changes in $\log _{e} F_{0}(t)$ from the phrase commands is named "phrase control mechanism' and its outputs are named 'phrase components.' Likewise, the mechanism that produces changes in $\log _{e} F_{0}(t)$ from the accent commands is named 'accent control mechanism' and its outputs are named 'accent components.' The outputs of these two mechanisms are added to a constant component $\log _{e} F_{b}$ to produce the final $\log _{e} F_{0}(t)$. Although a further mechanism ('glottal oscillation mechanism') is required to obtain the glottal source waveform, this final stage can be disregarded in the discussion of $\log _{e} F_{0}(t)$. For the rest of the paper, we shall use the word ' $F_{0}$-contour' to indicate $\log _{e} F_{0}(t)$.

In this model, the $F_{0}$ contour is expressed by

$$
\begin{gathered}
\log _{e} F_{0}(t)=\log _{e} F_{b}+\sum_{i=1}^{I} A p_{i} G p\left(t-T_{0 i}\right) \\
+\sum_{j=1}^{J} A a_{j}\left\{G a\left(t-T_{1 j}\right)-G a\left(t-T_{2 j}\right)\right\}, \\
G p(t)= \begin{cases}\alpha^{2} t \exp (-\alpha t), & \text { for } t \geq 0, \\
0, & \text { for } t<0,\end{cases} \\
G a(t)= \begin{cases}\min [1-(1+\beta t) \exp (-\beta t), \gamma], & \text { for } t \geq 0, \\
0, & \text { for } t<0,\end{cases}
\end{gathered}
$$

where $G p(t)$ represents the impulse response function of the phrase control mechanism and $G a(t)$ represents the step response function of the accent control mechanism.

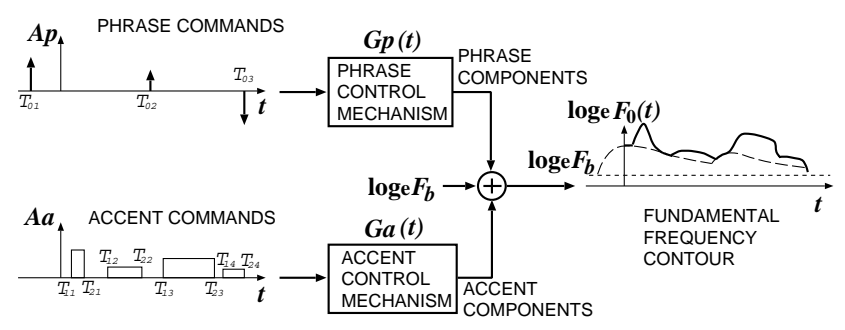

Fig. 1. A functional model for the process of generating $F_{0}$ contours. 
The symbols in these equations indicate

$F_{b}$ : baseline value of fundamental frequency,

$I$ : number of phrase commands,

$J$ : number of accent commands,

$A p_{i}$ : magnitude of the $i$ th phrase command,

$A a_{j}$ : amplitude of the $j$ th accent command,

$T_{0 i}$ : timing of the $i$ th phrase command,

$T_{1 j}$ : onset of the $j$ th accent command,

$T_{2 j}$ : offset of the $j$ th accent command,

$\alpha$ : natural angular frequency of the phrase control mechanism,

$\beta$ : natural angular frequency of the accent control mechanism,

$\gamma$ : relative ceiling level of accent components.

Parameters $\alpha$ and $\beta$ are known to be almost constant within an utterance as well as across utterances of a particular speaker. Although certain individual differences exist across speakers, it has been shown that $\alpha=3.0$ and $\beta=20.0$ can be used as default values. Parameter $\gamma$ may be variable across utterances and speakers, but it has also been shown that $\gamma=0.9$ can be used as a default value.

\section{NECESSITY OF PIECEWISE SMOOTHING OF MEASURED $\boldsymbol{F}_{0}$ CONTOURS}

Since it is possible to use default values for $\alpha, \beta$, and $\gamma$, the inverse problem is reduced to finding good first-order approximations to the number, temporal locations (henceforth 'timing'), and magnitudes/amplitudes of the phrase/accent commands. The baseline frequency $F_{b}$ can be obtained automatically by minimizing the mean squared error between the measured $F_{0}$ contour and the model-generated $F_{0}$ contour.

Several attempts have already been reported toward automatic extraction of $F_{0}$ contour parameters using the above-mentioned model [2] - [7]. These approaches, however, have made only limited success. The major reason is that the actual $F_{0}$ contour contains a number of factors that are not covered by the model, such as (1) gross errors in the measurement of $F_{0},(2)$ local deviations due to microprosody caused by certain consonants, (3) discontinuities due to the presence of voiceless consonants and utterance-medial pauses, and (4) lack of smoothness (i.e., non-differentiability). For the reliable estimation of the first-order approximations of model parameters, therefore, it is necessary to cope with these factors.

Since temporal changes of phrase components are generally much more gradual than those of accent components, the inflection points of the $F_{0}$ contour will roughly correspond to those of the accent components, and hence to the onsets and offsets of the corresponding accent commands except for a delay of $1 / \beta[\mathrm{s}]$. If the measured $F_{0}$ contour is approximated by smooth curve consisting of third-order polynomial segments, its points of inflection can be obtained by taking the second derivative of each third-order polynomial segment and putting it equal to zero. Thus the problem is reduced to a trivial one of solving a linear equation.

Since the current approach [8] is based upon the combination of approaches adopted in [2] and [3], it is necessary to convert the measured $F_{0}$ contour of an utterance into a continuous curve consisting of third-order polynomial segments, in such a way that the resulting curve is differentiable everywhere. Once this is done, its points of inflection (i.e., points where the first derivative is at a maximum or a minimum) should indicate points that are closely related to the onset and offset of the accent commands with an approximately constant delay.

\section{PRE-PROCESSING OF MEASURED $\boldsymbol{F}_{0}$ CONTOURS}

Pre-processing of an actual $F_{0}$ contour consists of four stages: (1) gross error correction, (2) microprosody removal, (3) interpolation, and (4) smoothing.

\subsection{Correction of Gross Errors}

Due to irregularities inherent in the mechanism of vocal fold vibration, no existing algorithm is completely free from gross errors. Gross errors can be classified into two types: (1) assignment of a false value (including zero) to a frame corresponding to a voiced interval, and (2) assignment of non-zero frequency value to a frame corresponding to a voiceless interval. The algorithm for correction of these two types of gross errors consists of the following two stages:

\subsubsection{Correction of gross errors in voiced intervals}

If the total number of frames with non-zero $F_{0}$ values is larger than $2 m+1$, and if

$$
\left|\frac{\log _{e} F_{0}(i)}{\log _{e} F_{0}(M \mid i, 2 m+1)}-1\right|>S,
$$

where $F_{0}(M \mid i, 2 m+1)$ indicates the median value of $F_{0}$ over the $(2 m+1)$ frames centered at the $i$ th frame, then mark $F_{0}(i)$ to be a gross error. In all other cases, $F_{0}(i)$ is not regarded as a gross error.

After $F_{0}$ values of all the frames are thus examined, $F_{0}$ values judged to be gross errors are replaced by linear interpolation in the $\log _{e} F_{0}$ domain. For a frame step of $10 \mathrm{~ms}, m=2$ and $S=$ 0.01 were found to be appropriate on the basis of preliminary experiments.

\subsubsection{Correction of gross errors in silent or unvoiced intervals}

Since gross errors due to false detection of $F_{0}$ in silent or unvoiced intervals seldom occur in successive frames, they can be removed by median smoothing over $(2 n+1)$ frames. For a frame step of $10 \mathrm{~ms}, n=2$ was found to be appropriate, allowing the correction of gross errors in at most two consecutive unvoiced frames.

\subsection{Removal of Microprosody}

The influence of consonantal articulation on $F_{0}$ contours, called 'microprosody', is often quite large especially in voiceless consonants, and thus has to be removed, since it is not included in the model. It appears as $F_{0}$ transitions at boundaries between adjacent vowels. The procedure for removing the consonantal disturbances can be stated as follows.

Let $i$ be the frame number that immediately precedes the voiceless consonant and $j$ be the frame number that immediately follows it. Calculate the gradients of the $F_{0}$ contour (to be denoted by $\left.G_{0}(\cdot)\right)$ in the vicinity of these boundaries.

If, for $n_{1} \leq 10, G_{0}\left(i-n_{1}\right)$ has the same sign as $G_{0}(i-1)$ and $\left|G_{0}\left(i-n_{1}\right)\right|<\left|G_{0}(i-1)\right| / 2$, remove all $F_{0}$ data at frames $(i-$ $\left.n_{1}, i-n_{1}+1, \cdots, i-1, i\right)$. Likewise, for $n_{2} \leq 10$, if $G_{0}\left(j+n_{2}\right)$ has the same sign as $G_{0}(j+1)$ and $\left|G_{0}\left(j+n_{2}\right)\right|<\left|G_{0}(j+1)\right| / 2$, remove all $F_{0}$ data at frames $\left(j, j+1, \cdots, j+n_{2}-1, j+n_{2}\right)$. 


\subsection{Interpolation of Intervals of Voiceless Consonants}

After removal of $F_{0}$ data perturbed by microprosody, the $F_{0}$ contour for the interval including the original voiceless consonant and the microprosodic sections is interpolated by the following procedure.

By re-defining the starting and ending frame numbers of the interval to be interpolated as $[i+1, j-1]$, the $F_{0}$ contour for an expanded interval $[i-p, j+p]$ is approximated by a third order polynomial equation

$$
\log _{e} F_{0}(t)=a_{0}+a_{1} t+a_{2} t^{2}+a_{3} t^{3},
$$

whose coefficients $\left[a_{0}, a_{1}, a_{2}, a_{3}\right]$ are obtained by solving the following simultaneous equations:

$$
\left\{\begin{aligned}
F_{0}(i) & =a_{0}+a_{1} i+a_{2} i^{2}+a_{3} i^{3}, \\
G_{0}(i) & =a_{1}+2 a_{2} i+3 a_{3} i^{2}, \\
F_{0}(j) & =a_{0}+a_{1} j+a_{2} j^{2}+a_{3} j^{3}, \\
G_{0}(j) & =a_{1}+2 a_{2} j+3 a_{3} j^{2} .
\end{aligned}\right.
$$

This interpolation is performed for intervals whose lengths are less than $333(=1 / \alpha) \mathrm{ms}$ (i.e., for $j-i+1<34$ at a frame interval of 10ms). Longer intervals are considered as pauses and are not interpolated. The length of the adjacent 'voiced' interval at each end is selected to be $50 \mathrm{~ms}$ (i.e., $p=4$ at a frame interval of $10 \mathrm{~ms}$ ). This procedure assigns a continuous contour for the expanded 'voiceless' interval, but does not guarantee its continuity with the adjacent $F_{0}$ data.

\subsection{Smoothing}

The interpolated $F_{0}$ contour is further smoothed by the following procedures to obtain an approximation that is continuous and differentiable everywhere.

(1) For the first $150 \mathrm{~ms}$, the coefficients $\left[a_{0}, a_{1}, a_{2}, a_{3}\right]$ of the best third-order polynomial approximation (in the sense of the least mean squared error) are obtained by solving the following set of linear equations:

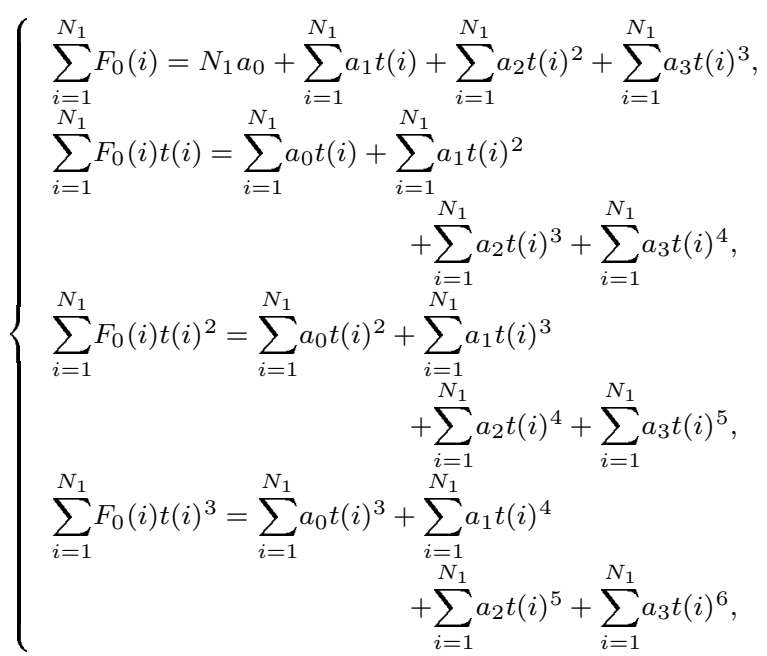

where $N_{1}$ indicates the number of frames within the initial interval of $200 \mathrm{~ms}\left(N_{1}\right.$ is equal to 20 at a frame interval of $10 \mathrm{~ms}$, but is smaller if the initial interval is shorter than $200 \mathrm{~ms}$ ).

(2) For the subsequent $150 \mathrm{~ms}$, the coefficients $\left[a_{0}, a_{1}, a_{2}, a_{3}\right]$ of the best third-order polynomial approximation are obtained with the additional constraint that the third-order polynomial should be continuous with the immediately preceding one both in amplitude and derivative, and are given by solving the following set of linear equations:

$$
\left\{\begin{array}{c}
F_{0}(j)=a_{0}+a_{1} t(j)+a_{2} t(j)^{2}+a_{3} t(j)^{3} \\
G_{0}(j)=a_{1}+2 a_{2} t(j)+3 a_{3} t(j)^{2}, \\
\sum_{j=1}^{N_{2}} F_{0}(j)=N_{2} a_{0}+\sum_{j=1}^{N_{2}} a_{1} t(j) \\
\quad+\sum_{j=1}^{N_{2}} a_{2} t(j)^{2}+\sum_{j=1}^{N_{2}} a_{3} t(j)^{3} \\
\sum_{j=1}^{N_{2}} F_{0}(j) t(j)=\sum_{j=1}^{N_{2}} a_{0} t(j)+\sum_{j=1}^{N_{2}} a_{1} t(j)^{2} \\
+\sum_{j=1}^{N_{2}} a_{2} t(j)^{3}+\sum_{j=1}^{N_{2}} a_{3} t(j)^{4}
\end{array}\right.
$$

where $j=1$ corresponds to $i=3 N_{1} / 4$ and $N_{2}$ indicates the number of frames within the current interval of $200 \mathrm{~ms}$. This procedure is repeated within a segment of utterance delimited by two adjacent pauses.

These procedures can give an approximation to the original $F_{0}$ contour consisting of piecewise third-order polynomial segments that are continuous and differentiable everywhere except for pause intervals.

\section{DERIVATION OF THE FIRST-ORDER APPROXIMATIONS OF COMMAND PARAMETERS}

\subsection{Extraction of accent command parameters}

Since the final outcome of smoothing is continuous and differentiable everywhere, it is quite straightforward to compute its derivative and find its maxima and minima analytically. If we neglect the effects of phrase components, the maxima and the minima of the first derivative of the contour should correspond to the onset and the offsets of accent commands with a constant delay of $1 / \beta$. The actual procedure is to detect the largest maximum and the smallest minimum for each interval where the sign of the derivative remains the same. A pair of maximum and minimum thus corresponds to the onset and the offset of an accent command. The mean absolute amplitudes of such a pair of a maximum and a minimum can be adopted as the first-order approximation to the amplitude of the corresponding accent command. If the initial part of the first-order derivative is negative and gives a minimum, then it can be regarded as the offset of the utterance-initial accent command, in which case one has to assume the existence of onset of the accent command before the start of an utterance.

\subsection{Extraction of phrase command parameters}

After approximately removing the accent components from the smoothed $F_{0}$ contour, one obtains a residual contour which consists mainly of phrase components. Since the influence of each phrase command is essentially a semi-infinite function of time starting from the onset of the command, each phrase command is detected successively by a left-to-right procedure from the residual contour. 


\section{EXPERIMENT}

\subsection{The Speech Material}

The speech material for the present study was a 15-minute recording of a male announcer's speech from a radio program "From My Bookshelf." It is a reading of a book. The speech signal was digitized at $10 \mathrm{kHz}$ with 16-bit precision, and the fundamental frequency was extracted by a modified autocorrelation analysis of the LPC residual signal.

\subsection{Results}

Figure 2 illustrates an example of the speech waveform and the results of successive processing for the Japanese utterance: "Ikutsukano otodake sokokara shakuyooshite, raibuno fun'ikio sokonawazuni henshuusuru kotoga dekiru." (The recording of the concert can be edited without destroying the impression of a live performance, by borrowing only a few sounds from the rehearsal.)

(1) Text and speech waveform

"Ikutsukano otodake sokokara shakuyooshite,

raibuno fun'ikio sokonawazuni henshuusuru kotoga dekiru."

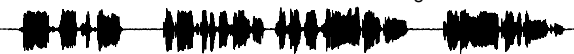

(2) Measured $F_{0}$ contour

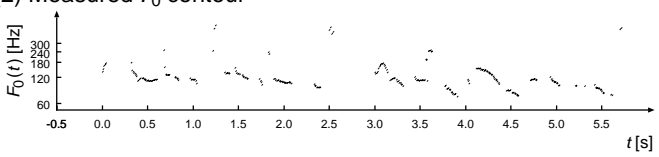

(3) After gross error correction

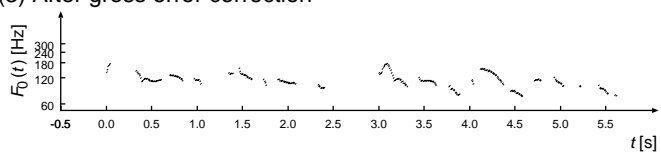

(4) After microprosody removal

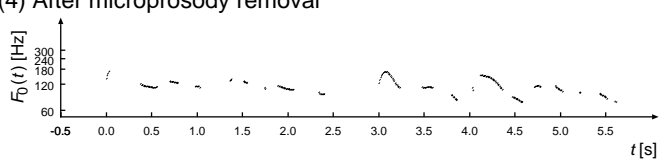

(5) Smoothed $F_{0}$ contour

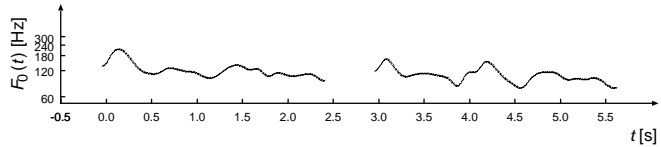

(6) Derivative

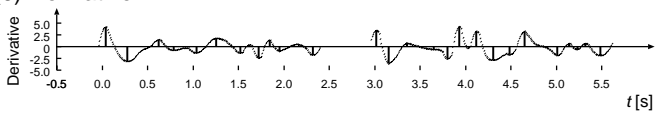

(7) First-order approximations of accent commands

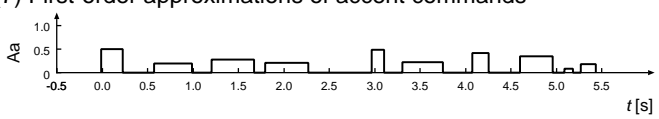

(8) First-order approximations of phrase commands

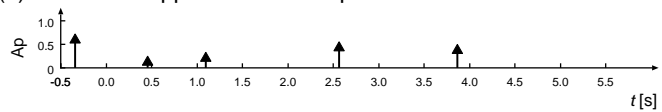

(9) First-order approximation to the smoothed $F_{0}$ contour

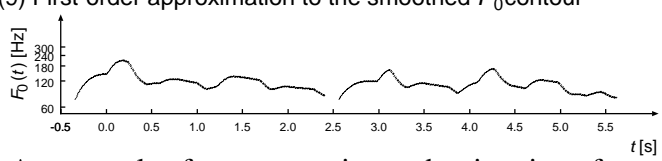

Fig. 2. An example of pre-processing and estimation of commands from an $F_{0}$ contour.
The figure shows, from top to bottom, (1) the speech waveform, (2) the measured $F_{0}$ contour, (3) the contour after gross error correction, (4) the contour after microprosody removal, (5) the contour after smoothing, (6) the derivative of the smoothed contour, (7) first-order approximations of accent commands, (8) first-order approximations of phrase commands, and (9) the first-order approximation to the smoothed $F_{0}$ contour. These panels clearly indicate that the method can give very good first-order approximations for the accent commands, and is therefore quite useful as the initial stage of fully automatic estimation of $F_{0}$ contour parameters.

Results of experiments on a total of 85 utterances indicate that out of 823 gross errors 815 were corrected, and out of $563 \mathrm{mi}-$ croprosodic disturbances 509 were removed. Thus the automatic correction was successfully performed on more than $95 \%$ of all the gross errors and microprosodic disturbances.

The results of automatic extraction of the phrase and accent components were then compared with those of manual analysis by an experienced researcher. Assuming that the results of manual analysis are $100 \%$ correct, the rate of correct extraction was about $90 \%$ for the accent commands, and was about $84 \%$ for the phrase commands.

\section{CONCLUSIONS}

The present paper has described our on-going work toward fully automatic extraction of $F_{0}$ contour parameters. We have shown that the inverse problem of deriving the commands from a measured $F_{0}$ contour can be converted into an analytically solvable problem, by approximating a measured $F_{0}$ contour by a smooth curve consisting of third-order polynomial segments that are continuous and differentiable everywhere except for pause intervals. Experimental results have shown the validity of the approach, but have also indicated the need for further work.

\section{REFERENCES}

[1] H. Fujisaki and S. Nagashima, "A model for synthesis of pitch contours of connected speech," Annual Report, Engg. Res. Inst., University of Tokyo, vol. 28, pp. 53-60 (1969).

[2] H. Fujisaki, K. Hirose and S. Seto, "A study on automatic extraction of characteristic parameters of fundamental frequency contours," Proc. Fall Meeting, Acoust. Soc. Jpn., vol. 1, pp. 255-256 (1990).

[3] E. Geoffrois, "A pitch contour analysis guided by prosodic event detection," Proc. Eurospeech '93, Berlin, vol. 2, pp. 793-796 (1993).

[4] H. Fujisaki, S. Ohno and Y. Wada, "A method for automatic estimation of parameters of a model for the generation process of fundamental frequency contours of speech," Proc. Spring Meeting, Acoust. Soc. Jpn., vol. 1, pp. 17-18 (1995).

[5] H. Fujisaki, S. Ohno and O. Tomita, "Automatic parameter extraction of fundamental frequency contours of speech based on a generative model," Proc. ICSP'96, Beijing, vol. 1, pp. 729-732 (1996).

[6] J. Mersdorf, A. Rinscheid, M. Brúggen and K. W. Schmidt, "Coding of large intonational units by linear prediction," Proc. ESCA Workshop on Intonation, Athens, pp. 235-238 (1997).

[7] H. Mixdorff, "A novel approach to the fully automatic extraction of fujisaki model parameters," Proc. ICASSP 2000. Istanbul, vol. 3, pp. 1281-1284 (2000).

[8] S. Narusawa, N. Minematsu, K. Hirose and H. Fujisaki, "Automatic extraction of parameters from fundamental frequency contours of speech," Proc. ICSP 2001, Seoul, vol. 2, pp. 833-838 (2001). 